Mathematical Research Letters 2, 79-94 (1995)

\title{
HARMONIC FUNCTIONS OF LINEAR GROWTH ON KÄHLER MANIFOLDS WITH NONNEGATIVE RICCI CURVATURE
}

\author{
Peter Li
}

\section{Introduction}

The subject began in 1975, when Yau [Y1] proved that there are no nonconstant, positive harmonic functions on a complete manifold with nonnegative Ricci curvature. A few years later, Cheng [C] pointed out that using a local version of Yau's gradient estimate, developed in his joint work with Yau $[\mathrm{CY}]$, one can show that there are no nonconstant harmonic functions of sublinear growth on a manifold with nonnegative Ricci curvature. Using Euclidean space as a model, this prompted Yau [Y3] to suggest in his 1981 IMU lectures the study of the space of harmonic functions of polynomial growth on manifolds with nonnegative Ricci curvature. He later conjectured that the space of harmonic functions which grows at most polynomially of order $p$, for $p \in \mathbb{Z}^{+}$, on a complete manifold with nonnegative Ricci curvature is finite dimensional. Part of this conjecture was verified by Tam and the author in [TL1]. In fact, they proved a sharp estimate on the dimension of the space of harmonic functions of linear growth. To state this theorem in a precise form, let us first make the following definition:

Definition. Let $\mathcal{H}^{p}(M)$ be the space of harmonic functions defined on $M$, satisfying the growth condition

$$
|f(x)|=O\left(r_{x_{0}}^{p}(x)\right)
$$

where $r_{x_{0}}(x)$ is the distance function to some fixed point $x_{0} \in M$.

Received December 5, 1994.

Research partially support by NSF grant DMS9300422. 
Theorem A (Li-Tam). Let $M^{n}$ be a complete manifold with nonnegative Ricci curvature. Suppose $x_{0} \in M$ is a fixed point and there exists a constant $k>0$ such that the volume of the geodesic ball centered at $x_{0}$ of radius $r$ satisfies

$$
V_{x_{0}}(r)=O\left(r^{k}\right)
$$

as $r \rightarrow \infty$. Then the dimension of $\mathcal{H}^{1}(M)$ satisfies the estimate

$$
\operatorname{dim} \mathcal{H}^{1}(M) \leq k+1 .
$$

Note that the standard Bishop volume comparison theorem implies that $k \leq n$. Also a theorem of Yau [Y2] and Cheeger-Gromov-Taylor [CGT] implies that $1 \leq k$.

Let us also point out that $\mathcal{H}^{1}\left(\mathbb{R}^{k}\right)=k+1$. Indeed, a basis for $\mathcal{H}^{1}\left(\mathbb{R}^{k}\right)$ is given by the constant function 1 and the standard rectangular coordinate functions. Using this, we can rewrite the Li-Tam estimate as

$$
\operatorname{dim} \mathcal{H}^{1}(M) \leq \operatorname{dim} \mathcal{H}^{1}\left(\mathbb{R}^{k}\right) .
$$

A natural question to ask, and it should be viewed as a refinement of Yau's conjecture, is as follows:

Question. Let $M$ be a complete manifold with nonnegative Ricci curvature. Suppose

$$
V_{x_{0}}(r)=O\left(r^{k}\right)
$$

Is it true that

$$
\operatorname{dim} \mathcal{H}^{p}(M) \leq \operatorname{dim} \mathcal{H}^{p}\left(\mathbb{R}^{k}\right)
$$

for all nonnegative integer $p$ ?

There are some partial results in the affirmative direction of this question. Tam and the author in [LT2] studied complete surfaces with finite total curvature. In particular, they gave relatively sharp upper and lower bounds for the dimension of $\mathcal{H}^{p}(M)$. When restricted to surfaces with nonnegative Gaussian curvature, their estimates confirmed inequality (1). In fact, the upper and lower bounds for the dimension of $\mathcal{H}^{p}(M)$ coincide when the surface has nonnegative Gaussian curvature outside a compact set. The interested reader should refer to [LT2] for a detailed statement of the theorem. We would also like to mention that Kasue [K1] also independently proved the upper bound for $\operatorname{dim} \mathcal{H}^{p}(M)$ on surfaces with finite total curvature. In some later work [K2,K3], Kasue also considered arbitrary $n$-dimensional complete manifolds that are in one of the following classes:

(i) The sectional curvature of $M$ satisfies $\left|K_{M}\right|(x) \leq C_{1} r_{x_{0}}^{-2-\epsilon}(x)$, and the volume growth at each end $E$ satisfies $V\left(E \cap B_{x_{0}}(r)\right) \geq C_{2} r^{n}$. 
(ii) $M$ is a simply connected manifold with sectional curvature bounded by $-C_{1} r_{x_{0}}^{-2}(x) \leq K_{M}(x) \leq 0$, and the volume growth satisfies $V_{x_{0}}(r) \geq C_{2} r^{n}$.

(iii) The sectional curvature satisfies $0 \leq K_{M}$, and the volume growth satisfies $V_{x_{0}}(r) \geq C_{2} r^{n}$.

(iv) The sectional curvature is bounded by $0 \leq K_{M}(x) \leq C_{1} r_{x_{0}}^{-2}(x)$ for all $x \in M$.

The constants above $-C_{1}, C_{2}$, and $\epsilon$-are assumed to be nonnegative. In those situations, he showed that $\operatorname{dim} \mathcal{H}^{p}(M)<\infty$. The arguments of Kasue used the theory of weighted Sobolev spaces. This method is effective in the case when the manifold is in some sense asymptotically Euclidean. However, when the assumption is on Ricci curvature or that the curvature is not asymptotically flat at infinity, the theory of weighted Sobolev spaces is unavailable in sufficient effectiveness to be suited for our purpose.

Recently, a more interesting development in this direction was established by Wang [W]. He proved that if $M$ is a complete manifold with nonnegative Ricci curvature outside a compact set $D$, and if its first Betti number is finite, then there exists a constant $0<C<\infty$ such that $\operatorname{dim} \mathcal{H}^{1}(M) \leq C$. Moreover, the constant $C$ only depends on the dimension, the diameter of $D$, and the lower bound of the Ricci curvature of $D$. Wang utilized the type of argument used in [LT1] and combined with the estimate on the Green's function developed in [LT3]. It was in [LT3] where the assumption on the Betti number is required. The intriguing phenomenon in Wang's theorem is that it is possible for $M$ to have nonconstant harmonic functions of sublinear growth. In fact, one can consider the metric

$$
d s^{2}=d r^{2}+f(r) r^{2} d \theta^{2}
$$

on $\mathbb{R}^{n}$, where $d \theta^{2}$ is the standard metric on the unit $(n-1)$-sphere and $f(r)$ is a smooth function satisfying $f(r)=1$ when $r$ is close to 0 , and $f(r)=\alpha$ when $r$ is sufficiently large. This metric is flat outside a compact set. However, using separation of variables, one can determine that for any fixed real number $p>0, \operatorname{dim} \mathcal{H}^{p}(M)$ is a nondecreasing function of $\alpha$. In fact, $\operatorname{dim} \mathcal{H}^{p}(M) \rightarrow \infty$ as $\alpha \rightarrow \infty$.

The purpose of this paper is to study the equality case of Theorem A. Unfortunately, we still cannot characterize those manifolds with nonnegative Ricci curvature that has $\operatorname{dim} \mathcal{H}^{1}(M)=n+1$. However, if we pass to the Kähler category, then we can prove that such a manifold is necessarily $\mathbb{C}^{m}$ with $2 m=n$.

Theorem 1. Let $M^{m}$ be a complete Kähler manifold with nonnegative Ricci curvature. Then $\operatorname{dim} \mathcal{H}^{1}(M) \leq 2 m+1$. Moreover, if $\operatorname{dim} \mathcal{H}^{1}(M)=$ $2 m+1$ then $M$ is isometric to $\mathbb{C}^{m}$ with the standard flat metric. 
During the course of the proof of Theorem 1, we will point out when the Kähler assumption is being used. It is conceivable that this is just a technical reason and that a theorem in the same spirit as Theorem 1 is true for the Riemannian case. Throughout this paper, we will denote the complex dimension of the Kähler manifold $M$ by $m$, and the real dimension of $M$ by $n$ if $M$ is not assumed to be complex.

\section{Outline of the proof of Theorem A}

In order to prove Theorem 1, it is necessary for us to recall some of the arguments which were used in the proof of Theorem A. The first key ingredient is the following lemma which was proved by the author in [L].

Lemma B. Let $M^{n}$ be a complete Riemannian manifold with nonnegative Ricci curvature. If $h$ is a bounded subharmonic function defined on $M$, then $h$ satisfies

$$
\lim _{r \rightarrow \infty} V_{x_{0}}^{-1}(r) \int_{B_{x_{0}}(r)} h(y) d y=\sup _{y \in M} h(y)
$$

To prove Theorem A, the authors of [LT1] considered the following two disjoint situations. The easy case is when the manifold has only small ends, which means that

$$
\int_{0}^{\infty} \frac{t d t}{V_{x_{0}}(t)}=\infty
$$

Together with the assumption that $M$ has nonnegative Ricci curvature, this volume growth condition is also equivalent to the condition that $M$ is parabolic (see $[\mathrm{V}]$ and $[\mathrm{LY}]$ ), i.e., $M$ does not admit a positive Green's function. The equivalence of parabolicity and small ends can be extended to a larger class of manifolds with weaker curvature assumption. The interested reader should refer to [LT3] for a more updated theorem in this direction.

Let $f$ be a harmonic function of linear growth on $M$. Since $M$ has nonnegative Ricci curvature, the local gradient estimate of Cheng-Yau [CY] implies that $|\nabla f|^{2}$ is bounded. However, Bochner's formula implies that $|\nabla f|^{2}$ is subharmonic on $M$. If $M$ satisfies (2), then it is known [LT2] that a bounded subharmonic function, hence $|\nabla f|^{2}$, must be constant. Applying Bochner's formula again, we can conclude that $M$ must isometrically split into a product manifold of the form $N \times \mathbb{R}$. The same type of argument will show that if $M$ satisfies (2) and has $\ell$ linearly independent, harmonic 
functions of linear growth, then $M$ must be a product manifold of the form $N \times \mathbb{R}^{\ell}$. However, condition (2) implies that $\ell \leq 2$, hence

$$
\operatorname{dim} \mathcal{H}^{1}(M) \leq 3
$$

If, in addition,

$$
V_{x_{0}}(r)=O(r)
$$

then $\ell \leq 1$ and

$$
\operatorname{dim} \mathcal{H}^{1}(M) \leq 2 .
$$

The complementary case is given by the condition

$$
\int_{0}^{\infty} \frac{t d t}{V_{x_{0}}(t)}<\infty
$$

which is equivalent to $M$ being nonparabolic. In this case, we use Lemma $\mathrm{B}$ to conclude that the bounded subharmonic function $|\nabla f|^{2}$ must satisfy

$$
\lim _{r \rightarrow \infty} V_{x_{0}}^{-1}(r) \int_{B_{x_{0}}(r)}|\nabla f|^{2}(y) d y=\sup _{y \in M}|\nabla f|^{2}(y) .
$$

This fact allows us to define an inner product given by

$$
\langle\langle f, g\rangle\rangle=\lim _{r \rightarrow \infty} V_{x_{0}}^{-1}(r) \int_{B_{x_{0}}(r)}\langle\nabla f, \nabla g\rangle(y) d y
$$

on the space

$$
\mathcal{H}_{0}^{1}(M)=\left\{f \mid f \in \mathcal{H}^{1}(M) \text { and } f\left(x_{0}\right)=0\right\}
$$

Using this inner product, one can choose $\left\{f_{1}, f_{2}, \cdots, f_{\ell}\right\}$ as an orthonormal basis for $\mathcal{H}_{0}^{1}(M)$. The second key ingredient is the fact that if we define the function

$$
\rho^{2}(y)=\sum_{i=1}^{\ell} f_{i}^{2}(y)
$$

with $\ell=\operatorname{dim} \mathcal{H}_{0}^{1}(M)$, then it satisfies the estimate that

$$
|\nabla \rho|(y) \leq 1
$$

Actually, it was only proved in [LT1] that

$$
\left|\nabla\left(\rho^{2}\right)\right|(y) \leq 2\left|\phi_{1}\right|(y) \leq 2 r_{0}(y)
$$


where $\phi_{1}$ is one of the elements in an appropriately chosen orthonormal basis. However, the argument implies (3) because

$$
\left|\phi_{1}\right|(y) \leq \rho(y) \text {. }
$$

To finish the proof of Theorem A, one can simply apply the divergence theorem and (4) to

$$
\begin{aligned}
2 \int_{B_{x_{0}}(r)}\left(\sum_{i=1}^{\ell}\left|\nabla f_{i}\right|^{2}\right) & =\int_{B_{x_{0}}(r)} \Delta\left(\rho^{2}\right) \\
& =\int_{\partial B_{x_{0}}(r)} \frac{\partial\left(\rho^{2}\right)}{\partial r} \\
& \leq 2 r A_{x_{0}}(r)
\end{aligned}
$$

where $A_{x_{0}}(r)$ is the area of the $\partial B_{x_{0}}(r)$. Dividing this inequality by $2 V_{x_{0}}(r)$ and letting $r \rightarrow \infty$, we conclude that

$$
\begin{aligned}
\ell & =\left\langle\left\langle f_{i}, f_{i}\right\rangle\right\rangle \\
& \leq \lim _{r \rightarrow \infty} \frac{r A_{x_{0}}(r)}{V_{x_{0}}(r)} .
\end{aligned}
$$

The estimate on $\ell$ follows by integrating with respect to $r$.

\section{The holomorphic case}

In this section, we will first prove Theorem 1 in the holomorphic category.

Theorem 2. Let $M^{m}$ be a complete Kähler manifold with nonnegative Ricci curvature. Suppose $M$ admits $m$ linearly independent holomorphic functions of linear growth, then $M$ must be isometric to $C^{m}$ with the standard flat metric.

Before we proof this theorem, let us established a key lemma which is valid for the Riemannian case as well.

Lemma 3. Let $M^{n}$ be a complete Riemannian manifold with nonnegative Ricci curvature, and $x_{0} \in M$ be a fixed point. If $\operatorname{dim} \mathcal{H}^{1}(M)=n+1$ and $\left\{f_{1}, \cdots f_{n}\right\}$ is an orthonormal basis for $\mathcal{H}_{0}^{1}(M)$, then the map $F: M \rightarrow \mathbb{R}^{n}$ given by

$$
F=\left(f_{1}, \cdots, f_{n}\right)
$$

is proper. Moreover, the function $\rho^{2}(x)=\sum_{i=1}^{n} f_{i}^{2}(x)$ has the asymptotic behavior

$$
\rho(x) r_{x_{0}}^{-1}(x) \rightarrow 1
$$


as $x \rightarrow \infty$.

Proof. Let us first observe that according to Cheng [C], the assumption that $\operatorname{dim} \mathcal{H}^{1}(M)=n+1$ implies that there are $n$ linearly independent harmonic functions of linear growth on $M$. As in the proof of Theorem A outlined in the previous section, but using (3) instead of (4), we have

$$
\begin{aligned}
V_{x_{0}}^{-1}(r) \int_{B_{x_{0}}(r)}\left(\sum_{i=1}^{n}\left|\nabla f_{i}\right|^{2}\right) & \leq V_{x_{0}}^{-1}(r) \int_{\partial B_{x_{0}}(r)} \rho \\
& =\frac{r A_{x_{0}}(r)}{V_{x_{0}}(r)}\left(A_{x_{0}}^{-1}(r) \int_{\partial B_{x_{0}}(r)} \rho r^{-1}\right) .
\end{aligned}
$$

We now claim that

$$
\frac{r A_{x_{0}}(r)}{V_{x_{0}}(r)} \leq n
$$

To see this, let us consider the integral

$$
\int_{B_{x_{0}}(r)}\left|\nabla r_{x_{0}}\right|^{2}=-\int_{B_{x_{0}}(r)} r_{x_{0}} \Delta r_{x_{0}}+\int_{\partial B_{x_{0}}(r)} r_{x_{0}} .
$$

Using $\left|\nabla r_{x_{0}}\right|=1$ and the comparison theorem, which asserts that

$$
\Delta r_{x_{0}} \leq \frac{n-1}{r_{x_{0}}}
$$

(7) implies that

$$
V_{x_{0}}(r) \geq-(n-1) V_{x_{0}}(r)+r A_{x_{0}}(r)
$$

which is equivalent to (6). Substituting (6) into (5) and taking $r \rightarrow \infty$, we conclude that

$$
n=\sum_{i=1}^{n}\left\langle\left\langle f_{i}, f_{i}\right\rangle\right\rangle \leq n \lim _{r \rightarrow \infty}\left(A_{x_{0}}^{-1}(r) \int_{\partial B_{x_{0}}(r)} \rho r^{-1}\right) .
$$

However, using the fact that $\rho^{2}\left(x_{0}\right)=\sum_{i=1}^{n} f_{i}^{2}\left(x_{0}\right)=0$ and integrating (4) along a geodesic joining $x_{0}$ to $x$, we conclude that $\rho(x) r_{x_{0}}^{-1}(x) \leq 1$ as 
$x \rightarrow \infty$. This implies that for any given $\epsilon>0$, there exists $R(\epsilon)>0$ such that

$$
(1-\epsilon) A_{x_{0}}(r) \leq \int_{\partial B_{x_{0}}(r)} \rho r^{-1} \leq A_{x_{0}}(r)
$$

for all $r \geq R(\epsilon)$. Integrating from $R_{1}$ to $R_{2}$, for $R(\epsilon) \leq R_{1} \leq R_{2}$, we have

$$
(1-\epsilon) V_{x_{0}}\left(R_{1}, R_{2}\right) \leq \int_{B_{x_{0}}\left(R_{1}, R_{2}\right)} \rho r_{x_{0}}^{-1} \leq V_{x_{0}}\left(R_{1}, R_{2}\right)
$$

where $B_{x_{0}}\left(R_{1}, R_{2}\right)=B_{x_{0}}\left(R_{2}\right) \backslash B_{x_{0}}\left(R_{1}\right)$ and $V_{x_{0}}\left(R_{1}, R_{2}\right)=V_{x_{0}}\left(R_{2}\right)-$ $V_{x_{0}}\left(R_{1}\right)$.

For any $0<\delta<1$, let us define $m_{\delta}\left(R_{1}, R_{2}\right)$ to be the measure of the set

$$
\left\{x \in B_{x_{0}}\left(R_{1}, R_{2}\right) \mid \rho r_{x_{0}}^{-1} \leq 1-\delta\right\} .
$$

Clearly

$$
\int_{B_{x_{0}}\left(R_{1}, R_{2}\right)} \rho r_{x_{0}}^{-1} \leq(1-\delta) m_{\delta}\left(R_{1}, R_{2}\right)+\left(V_{x_{0}}\left(R_{1}, R_{2}\right)-m_{\delta}\left(R_{1}, R_{2}\right)\right) .
$$

Hence, together with (8), we conclude that

$$
(1-\epsilon) V_{x_{0}}\left(R_{1}, R_{2}\right) \leq(1-\delta) m_{\delta}\left(R_{1}, R_{2}\right)+\left(V_{x_{0}}\left(R_{1}, R_{2}\right)-m_{\delta}\left(R_{1}, R_{2}\right)\right)
$$

which implies that

$$
\frac{m_{\delta}\left(R_{1}, R_{2}\right)}{V_{x_{0}}\left(R_{1}, R_{2}\right)} \leq \frac{\epsilon}{\delta}
$$

Since $\epsilon$ is arbitrary, this proves that

$$
\frac{m_{\delta}\left(R_{1}, R_{2}\right)}{V_{x_{0}}\left(R_{1}, R_{2}\right)} \rightarrow 0
$$

as $R_{1}, R_{2} \rightarrow \infty$.

The estimate (3) implies that for any pair of points $x, y \in M$,

$$
\rho(y) \leq \rho(x)+r(x, y)
$$

where $r(x, y)$ is the geodesic distance between $x$ and $y$. This is equivalent to

$$
\rho(y) r_{x_{0}}^{-1}(y) \leq\left(\frac{\rho(x)}{r_{x_{0}}(y)}+\frac{r(x, y)}{r_{x_{0}}(y)}\right) .
$$


If there exists $\alpha>0$ and a sequence of points $x_{i} \in M$ such that $x_{i} \rightarrow \infty$ and

$$
\rho\left(x_{i}\right) r_{x_{0}}^{-1}\left(x_{i}\right) \leq(1-\alpha),
$$

then for any $y \in B_{x_{i}}\left(\frac{\alpha r_{x_{0}}\left(x_{i}\right)}{4}\right)$, we have

$$
\begin{aligned}
\rho(y) r_{x_{0}}^{-1}(y) & \leq\left(\frac{\rho\left(x_{i}\right)}{r_{x_{0}}(y)}+\frac{r\left(x_{i}, y\right)}{r_{x_{0}}(y)}\right) \\
& \leq\left(\frac{(1-\alpha) r_{x_{0}}\left(x_{i}\right)}{r_{x_{0}}(y)}+\frac{\alpha r_{x_{0}}\left(x_{i}\right)}{4 r_{x_{0}}(y)}\right)
\end{aligned}
$$

However, the triangle inequality implies that

$$
r_{x_{0}}(y)+\frac{\alpha r_{x_{0}}\left(x_{i}\right)}{4} \geq r_{x_{0}}(y)+r\left(x_{i}, y\right) \geq r_{x_{0}}\left(x_{i}\right) .
$$

Hence combining with (10), we have

$$
\rho(y) r_{x_{0}}^{-1}(y) \leq\left(\frac{4(1-\alpha)}{4-\alpha}+\frac{\alpha}{4-\alpha}\right)=\left(\frac{4-3 \alpha}{4-\alpha}\right)<1 .
$$

Let us set $\delta$ to satisfy

$$
1-\delta=\left(\frac{4-3 \alpha}{4-\alpha}\right)
$$

then

$$
\rho r_{x_{0}}^{-1} \leq 1-\delta
$$

on $B_{x_{i}}\left(\frac{\alpha r_{x_{0}}\left(x_{i}\right)}{4}\right)$. Obviously,

$$
V_{x_{i}}\left(\frac{\alpha r_{x_{0}}\left(x_{i}\right)}{4}\right) \leq m_{\delta}\left(\left(1-\frac{\alpha}{4}\right) r_{x_{0}}\left(x_{i}\right),\left(1+\frac{\alpha}{4}\right) r_{x_{0}}\left(x_{i}\right)\right) .
$$

On the other hand, the fact that

$$
B_{x_{0}}\left(\left(1+\frac{\alpha}{4}\right) r_{x_{0}}\left(x_{i}\right)\right) \subset B_{x_{i}}\left(\left(2+\frac{\alpha}{4}\right) r_{x_{0}}\left(x_{i}\right)\right)
$$

and the volume comparison theorem imply that

$$
\begin{aligned}
V_{x_{i}}\left(\frac{\alpha r_{x_{0}}\left(x_{i}\right)}{4}\right) & \geq V_{x_{i}}\left(\left(2+\frac{\alpha}{4}\right) r_{x_{0}}\left(x_{i}\right)\right)\left(\frac{\alpha}{4}\right)^{n}\left(2+\frac{\alpha}{4}\right)^{-n} \\
& \geq V_{x_{0}}\left(\left(1-\frac{\alpha}{4}\right) r_{x_{0}}\left(x_{i}\right),\left(1+\frac{\alpha}{4}\right) r_{x_{0}}\left(x_{i}\right)\right)\left(\frac{\alpha}{4}\right)^{n}\left(2+\frac{\alpha}{4}\right)^{-n}
\end{aligned}
$$


Combining with (11), we conclude that

$$
\frac{m_{\delta}\left(\left(1-\frac{\alpha}{4}\right) r_{x_{0}}\left(x_{i}\right),\left(1+\frac{\alpha}{4}\right) r_{x_{0}}\left(x_{i}\right)\right)}{V_{x_{0}}\left(\left(1-\frac{\alpha}{4}\right) r_{x_{0}}\left(x_{i}\right),\left(1+\frac{\alpha}{4}\right) r_{x_{0}}\left(x_{i}\right)\right)} \geq\left(\frac{\alpha}{4}\right)^{n}\left(2+\frac{\alpha}{4}\right)^{-n} .
$$

This contradicts (9) as $i \rightarrow \infty$, and we have established the claim that

$$
\rho(x) r_{x_{0}}^{-1}(x) \rightarrow 1
$$

as $x \rightarrow \infty$.

We are now ready to prove Theorem 2 .

Proof of Theorem 2. Let $\left\{g_{1}, \cdots, g_{m}\right\}$ be $m$ linearly independent holomorphic functions of linear growth. Since $g_{i}$ is of linear growth, we claim that the real part $\Re\left(g_{i}\right)$ and the imaginary part $\Im\left(g_{i}\right)$ are of linear growth. This is indeed the case; since both $\Re\left(g_{i}\right)$ and $\Im\left(g_{i}\right)$ are harmonic functions on $M$ that grow at most linearly, they are either of linear growth or identically constant. However, if one of them is constant then the other must also be constant because of the Cauchy-Riemann equations. This violates the fact that $g_{i}$ is of linear growth.

Moreover, the Cauchy-Riemann equations also imply that

$$
\left\langle\Re\left(g_{i}\right), \Im\left(g_{i}\right)\right\rangle \equiv 0
$$

and

$$
\left|\nabla \Re\left(g_{i}\right)\right| \equiv\left|\nabla \Im\left(g_{i}\right)\right| .
$$

Hence

$$
\left\langle\left\langle\Re\left(g_{i}\right), \Im\left(g_{i}\right)\right\rangle\right\rangle=0
$$

and

$$
\left\langle\left\langle\Re\left(g_{i}\right), \Re\left(g_{i}\right)\right\rangle\right\rangle=\left\langle\left\langle\Im\left(g_{i}\right), \Im\left(g_{i}\right)\right\rangle\right\rangle .
$$

Therefore, by a unitary change of basis, we may assume that the set

$$
\left\{\Re\left(g_{1}\right), \Im\left(g_{1}\right), \cdots, \Re\left(g_{m}\right), \Im\left(g_{m}\right)\right\}
$$

forms an orthonormal basis with respect to the inner product $\langle\langle\cdot, \cdot\rangle\rangle$. According to Lemma 4, the holomorphic map $G: M \rightarrow \mathbb{C}^{m}$ given by $G=$ $\left(g_{1}, \cdots, g_{m}\right)$ is proper and the function

$$
\rho^{2}(x)=\sum_{i=1}^{m}\left|g_{i}\right|^{2}
$$


satisfies

$$
\rho(x) r_{x_{0}}^{-1}(x) \rightarrow 1 .
$$

Let us set $f_{2 i-1}=\Re\left(g_{i}\right)$ and $f_{2 i}=\Im\left(g_{i}\right)$ for $i=1, \cdots, m$. Then the map $G=F=\left(f_{1}, \cdots, f_{2 m}\right)$ can be viewed as a map into $\mathbb{R}^{2 m}$. If we denote the differential of $F$ by $d F$, then the $p q$-th entry of the matrix $(d F) \circ(d F)^{t}$ is given by

$$
\sum_{\alpha=1}^{2 m} \frac{\partial f_{p}}{\partial z_{\alpha}} \frac{\partial f_{q}}{\partial z_{\alpha}}=\left\langle d f_{p}, d f_{q}\right\rangle .
$$

In this notation, the fact that $\left\{f_{1}, \cdots, f_{2 m}\right\}$ forms an orthonormal basis means that

$$
\lim _{r \rightarrow \infty} V_{x_{0}}^{-1}(r) \int_{B_{x_{0}}(r)} d F \circ(d F)^{t}=I .
$$

This implies that the integrand must be nonsingular somewhere and hence the differential $d F$ is nonsingular somewhere. Since the map $G$ is holomorphic and it is nonsingular at least at a point, the image $G(M)$ must be a subvariety of dimension $m$ in $\mathbb{C}^{m}$. By the properness of $G$, we conclude that $G$ must be surjective. Let us point out that this is the only part of the argument where holomorphicity is being used.

Using orthonormality of the basis $\left\{f_{1}, \cdots, f_{2 m}\right\}$ once again, and using the fact that the inner product

$$
\left\langle\left\langle f_{p}, f_{p}\right\rangle\right\rangle=\sup _{M}\left|\nabla f_{p}\right|^{2},
$$

we conclude that the energy density $e(F)$ of the map $F$ is bounded by

$$
e(F) \leq \sum_{p=1}^{2 m}\left|\nabla f_{p}\right|^{2} \leq 2 m
$$

However, the arithmetic-geometric mean implies that

$$
\frac{e(F)}{2 m} \geq(\operatorname{det}(d F))^{\frac{1}{2 m}} .
$$

where $\operatorname{det}(d F)$ is the determinant of the differential $d F$. This implies that the Jacobian of the map $J(F)=(\operatorname{det}(d F))^{\frac{1}{2}}$ satisfies

$$
J(F) \leq 1 .
$$

For $0<R_{1}<R_{2}$, let $\bar{B}\left(R_{1}, R_{2}\right) \subset \mathbb{C}^{m}$ be the annulus of radius $R_{1}$ to $R_{2}$ centered at the origin. The set $F^{-1}\left(\bar{B}\left(R_{1}, R_{2}\right)\right)$ is given by

$$
F^{-1}\left(\bar{B}\left(R_{1}, R_{2}\right)\right)=\left\{x \in M \mid R_{1} \leq \rho \leq R_{2}\right\}
$$


Since $F$ is surjective, we have

$$
\int_{F^{-1}\left(\bar{B}\left(R_{1}, R_{2}\right)\right)} J(F)(x) d x \geq \bar{V}\left(R_{1}, R_{2}\right),
$$

where $\bar{V}\left(R_{1}, R_{2}\right)$ is the Euclidean volume of $\bar{B}\left(R_{1}, R_{2}\right)$. Using (12), we conclude that

$$
\operatorname{Vol}\left(F^{-1}\left(\bar{B}\left(R_{1}, R_{2}\right)\right)\right) \geq \bar{V}\left(R_{1}, R_{2}\right) .
$$

where $\operatorname{Vol}\left(F^{-1}\left(\bar{B}\left(R_{1}, R_{2}\right)\right)\right)$ is the volume of the set $F^{-1}\left(\bar{B}\left(R_{1}, R_{2}\right)\right)$.

On the other hand, Lemma 4 implies that for any $\epsilon>0$, there exists $R(\epsilon)>0$ such that

$$
(1-\epsilon) r_{x_{0}}(x) \leq \rho(x) \leq r_{x_{0}}(x)
$$

for $\rho(x) \geq R(\epsilon)$. If we take $R_{1} \geq R(\epsilon)$, then we have

$$
F^{-1}\left(\bar{B}\left(R_{1}, R_{2}\right)\right) \subset B_{x_{0}}\left(R_{1}, \frac{R_{2}}{1-\epsilon}\right) .
$$

Combining with (13), and substituting $R_{2}=(1-\epsilon) R$, we obtain

$$
V_{x_{0}}\left(R_{1}, R\right) \geq \bar{V}\left(R_{1},(1-\epsilon) R\right) .
$$

Hence, we have shown that as $R \rightarrow \infty$, the ratio

$$
\begin{aligned}
\lim _{R \rightarrow \infty} \frac{V_{x_{0}}(R)}{\bar{V}(R)} & =\lim _{R \rightarrow \infty} \frac{V_{x_{0}}\left(R_{1}\right)+V_{x_{0}}\left(R_{1}, R\right)}{\bar{V}\left(R_{1}\right)+\bar{V}\left(R_{1}, R\right)} \\
& \geq \lim _{R \rightarrow \infty} \frac{\bar{V}\left(R_{1},(1-\epsilon) R\right)}{\bar{V}\left(R_{1}, R\right)} \\
& =(1-\epsilon)^{2 m} .
\end{aligned}
$$

Since $\epsilon$ is arbitrary, we conclude that

$$
\lim _{R \rightarrow \infty} \frac{V_{x_{0}}(R)}{\bar{V}(R)} \geq 1
$$

However, the Bishop volume comparison theorem implies that

$$
\lim _{R \rightarrow \infty} \frac{V_{x_{0}}(R)}{\bar{V}(R)} \leq 1
$$

and equality holds if and only if $M$ is isometric to $\mathbb{R}^{2 m}$. This implies that the map $F$ is an isometry and Theorem 3 is proved. 


\section{Reduction to the holomorphic case}

Proof of Theorem 1. Let us first assume that $M$ is simply connected. In this case, one needs to show that if $M$ has $2 m$ linearly independent harmonic functions of linear growth, then it must have $m$ linearly independent, linear growth, holomorphic functions. The proof of Theorem 1 in [LY2] can be adopted to prove this claim. In fact, since $M$ has nonnegative Ricci curvature, the argument simplifies substantially by applying the mean value inequality of [LS]. For the sake of completeness, let us outline the argument for our present situation.

First or all, Lemma 1 of [LY2] implies that the complex Hessian of a harmonic function $f$ on $M$ must satisfy the Bochner-type formula

$$
\Delta\left|f_{i \bar{j}}\right|^{\frac{m-1}{m}} \geq 0 \text {. }
$$

On the other hand, the standard Bochner's formula implies that

$$
\Delta|\nabla f|^{2} \geq 2 f_{p q}^{2},
$$

where $f_{p q}$ is the real Hessian of $f$. Let us define the nonnegative cutoff function $\phi$ satisfying

$$
\phi(x)= \begin{cases}1 & \text { on } B_{x_{0}}(R) \\ 0 & \text { on } M \backslash B_{x_{0}}(2 R)\end{cases}
$$

with $|\nabla \phi| \leq C R^{-1}$ for some constant $C>0$. Multiplying (15) by $\phi^{2}$ and integrating over $M$, we have

$$
\int_{M} \phi^{2} \Delta|\nabla f|^{2} \geq 2 \int_{M} \phi^{2} f_{p q}^{2}
$$

However, integrating the left hand side by parts yields

$$
\begin{aligned}
\int_{M} \phi^{2} \Delta|\nabla f|^{2} & =-4 \int_{M} \phi|\nabla f|\langle\nabla \phi, \nabla|\nabla f|\rangle \\
& \leq 4 \int_{M}|\nabla \phi|^{2}|\nabla f|^{2}+\left.\int_{M} \phi^{2}|\nabla| \nabla f\right|^{2} \\
& \leq 4 \int_{M}|\nabla \phi|^{2}|\nabla f|^{2}+\int_{M} \phi^{2} f_{p q}^{2} .
\end{aligned}
$$

Therefore, we conclude that

$$
\int_{M} \phi^{2} f_{p q}^{2} \leq 4 \int_{M}|\nabla \phi|^{2}|\nabla f|^{2}
$$


The definition of $\phi$ implies that

$$
\begin{aligned}
\int_{B_{x_{0}}(R)}\left|f_{i \bar{j}}\right|^{2} & \leq \int_{B_{x_{0}}(R)} f_{p q}^{2} \\
& \leq C R^{-2} \int_{B_{x_{0}}(2 R)}|\nabla f|^{2} .
\end{aligned}
$$

Dividing through by $V_{x_{0}}(R)$ and using the fact that $|\nabla f|$ is bounded, we obtain

$$
V_{x_{0}}^{-1}(R) \int_{B_{x_{0}}(R)}\left|f_{i \bar{j}}\right|^{2} \leq C R^{-2} \frac{V_{x_{0}}(2 R)}{V_{x_{0}}(R)} .
$$

The Bishop volume comparison theorem now implies that the right hand side tends to 0 as $R \rightarrow \infty$. Hence we conclude that

$$
\lim _{R \rightarrow \infty} V_{x_{0}}^{-1}(R) \int_{B_{x_{0}}(R)}\left|f_{i \bar{j}}\right|^{2}=0 .
$$

Applying the mean value inequality of Li-Schoen [LS] and using (14), we deduce that $f_{i \bar{j}} \equiv 0$. Therefore, $f$ is a pluri-harmonic function on $M$. The assumption that $M$ is simply connected implies that $f$ is either a real or imaginary part of a linear growth holomorphic function $g$. If $\operatorname{dim} \mathcal{H}_{0}^{1}(M)=n$, then the argument used in the proof of the Main Theorem in [LY2] applies and we conclude that there are $m$ linearly independent holomorphic functions of linear growth on $M$. Theorem 2 now implies that $M$ is isometric to $\mathbb{C}^{m}$.

To delete the simply connectedness assumption on $M$, we observe that the lift of a harmonic function of linear growth to the universal covering $\tilde{M}$ of $M$ is also a harmonic function of linear growth. Indeed, if $\pi: \tilde{M} \rightarrow M$ is the universal covering map, then we can define the lift of a harmonic function of linear growth $f$ on $M$ by

$$
\tilde{f}(y)=f \circ \pi(y) .
$$

Again, let us normalize $f$ by setting $f\left(x_{0}\right)=0$. Let $\left\{y_{0}, \cdots, y_{i}, \cdots\right\}$ be the set of preimage points of $x_{0}$ under $\pi$. Then $\tilde{f}\left(y_{i}\right)=0$. For any $y \in \tilde{M}$, let $\gamma$ be a minimizing geodesic in $M$ joining $\pi(y)$ to $x_{0}$. Lifting $\gamma$ to a minimizing geodesic $\tilde{\gamma}$ joining $y$ to a $y_{i}$, we see that

$$
\begin{aligned}
\tilde{r}\left(y_{0}, y\right) & \geq \tilde{r}\left(y_{i}, y\right) \\
& =r_{x_{0}}(\pi(y)),
\end{aligned}
$$


where $\tilde{r}$ denotes the distance function on $\tilde{M}$. However, the assumption on $f$ implies that

$$
\begin{aligned}
|\tilde{f}(y)| & =|f(\pi(y))| \\
& \leq C r_{x_{0}}(\pi(y)) \\
& =C \tilde{r}\left(y_{0}, y\right),
\end{aligned}
$$

which implies that $\tilde{f}$ is also of linear growth.

If $\operatorname{dim} \mathcal{H}_{0}^{1}(M)=2 m$, then by lifting all the harmonic functions of linear growth, we see that $\operatorname{dim} \mathcal{H}_{0}^{1}(\tilde{M})=2 m$. Applying the argument for the simply connected case to $\tilde{M}$, we conclude that the map $\tilde{F}: \rightarrow \mathbb{C}^{m}$ given by

$$
\tilde{F}=\left(\tilde{f}_{1}, \cdots, \tilde{f}_{2 m}\right)
$$

is an isometry. Moreover, the set $\left\{\tilde{f}_{i}\right\}$ corresponds to the rectangular coordinate functions of $\mathbb{C}^{m}$. In particular, they must separate points in $\tilde{M}$. On the other hand, $\tilde{f}_{i}$ comes from lifting $f_{i}$, hence $M=\tilde{M}$.

In the above argument of showing that the harmonic function of linear growth $f$ is pluri-harmonic, we only need the fact that $|\nabla f|$ grows sublinearly. On the other hand, the gradient estimate of Cheng-Yau implies that if $f$ is of sub-quadratic growth, then $|\nabla f|$ is of sublinear growth. Hence we can summarize by the following:

Corollary 5. Let $M$ be a complete Kähler manifold with nonnegative Ricci curvature. If $f$ is a harmonic function of sub-quadratic growth defined on $M$, then $f$ is pluri-harmonic.

\section{Acknowledgement}

The author would like to thank Gang Tian and Shing Tung Yau for bringing up the question in the Kähler category. He would also like to thank Jingyi Chen, Luen-fai Tam, and Jiaping Wang for many valuable conversations and their interest in this work.

\section{References}

[CGT] J. Cheeger, M. Gromov and M. Taylor, Finite propagation speed, kernel estimates for functions of the Laplace operator, and the geometry of complete Riemannian manifolds, J. Diff. Geom. 17 (1983), 15-53.

[C] S. Y. Cheng, Liouville theorem for harmonic maps, Proc. Symp. Pure Math. 36 (1980), 147-151.

[CY] S. Y. Cheng and S. T. Yau, Differential equations on Riemannian manifolds and their geometric applications, Comm. Pure Appl. Math. 28 (1975), 333-354. 
[K1] A. Kasue, Recent Topics in Differential and Analytic Geometry, Adv. Stud. Pure Math., vol. 18, North-Holland, 1989.

[K2] , Differential Geometry: Partial Differential Equations on Manifolds, Proc. Symp. Pure Math. Part I, vol. 54, AMS, 1993, pp. 281-290.

[K3] , Harmonic functions of polynomial growth on complete manifolds II, preprint.

[L] P. Li, Large time behavior of the heat equation on complete manifolds with nonnegative Ricci curvature, Ann. Math. 124 (1986), 1-21.

[LS] P. Li and R. Schoen, $L^{p}$ and mean value properties of subharmonic functions on Riemannian manifolds, Acta Math. 153 (1984), 279-301.

[LT1] P. Li and L. F. Tam, Linear growth harmonic functions on a complete manifold, J. Diff. Geom. 29 (1989), 421-425.

[LT2] _ Complete surfaces with finite total curvature, J. Diff. Geom. 33 (1991), $139-168$.

[LT3] , Green's functions, harmonic functions, and volume comparison (to appear in J. Diff. Geom.).

[LY1] P. Li and S. T. Yau, On the parabolic kernel of the Schrödinger operator, Acta Math. 156 (1986), 153-201.

[LY2] Complex Geometry, Lecture notes in pure and applied mathematics, vol. 143 (Gen Komatsu and Yusuke Sakane,, ed.), Marcel Dekker, New YorkBasel-Hong Kong, 1992, pp. 131-143..

[V] N. Varopoulos, The Poisson kernel on positively curved manifolds, J. Funct. Anal. 44 (1981), 359-380.

[Y1] S. T. Yau, Harmonic functions on complete Riemannian manifolds, Comm. Pure Appl. Math. 28 (1975), 201-228.

[Y2] S Some function-theoretic properties of complete Riemannian manifolds and their applications to geometry, Indiana Math. J. 25 (1976), 659-670.

[Y3] Nonlinear Analysis in Geometry, L'Enseignement Mathématique, Série des Conférences de l'Union Mathématique Internationale, No. 8, SRO-KUNDIG, Genève, 1986.

Department of M athematics, University of California, Irvine, Irvine, Ca $92717-3875$

E-mail address: pli@math.uci.edu 